\title{
Scarred scalp reconstruction with a rectangular expander
}

\author{
Hyojeong Shin, \\ Jeonghwan Shin, \\ Jun Yong Lee \\ Department of Plastic and \\ Reconstructive Surgery, College of \\ Medicine, The Catholic University of \\ Korea, Seoul, Korea
}

\begin{abstract}
Scalp reconstruction is challenging because the scalp is inelastic, stiff, and has hair follicles. Tissue expansion offers aesthetically pleasing outcomes with minimal donor-site morbidity. However, in a scarred scalp, the extent of possible dissection for the expander insertion may be limited and surgeons must make use of the limited scalp tissue. We successfully reconstructed scarred scalps using rectangular expanders. This report presents two cases: a $4 \times 3 \mathrm{~cm}$ chronic defect with widespread scarring and osteomyelitis and an $11 \times 7.5 \mathrm{~cm}$ scar tissue following a skin graft. Tissue expanders were inserted in the subgaleal plane and were inflated by $195 \mathrm{~mL}$ and $400 \mathrm{~mL}$ over periods of 2 and 3 months, respectively. Subgaleal elevation of a fasciocutaneous flap was achieved with the expanded tissue. The defects were well covered, with good color, texture, and hair-bearing tissue. There were no complications involving the tissue expanders. Rectangular expanders yield more available tissue than round or crescent-shaped expanders. Moreover, since the base of the flap is well defined, the expander can be easily inserted in a limited space. Therefore, rectangular expanders are recommended for the reconstruction of scarred scalps.
\end{abstract}

Keywords: Cicatrix / Scalp / Tissue expanders

\section{INTRODUCTION}

Scalp reconstruction is challenging because the scalp is inelastic, stiff, and has hair follicles [1]. Cosmetic considerations are also relevant, as this zone makes a major contribution to individuals' appearance. If possible, local flaps are preferable for scalp reconstruction because they use original tissue, resulting in a natural appearance, which is especially relevant for hairbearing tissue. However, the applicability of local flaps can be limited in patients with defects that are larger than $50 \mathrm{~cm}^{2}$ or require extensive undermining [2], and excessive tension leads

Correspondence: Jun Yong Lee

Department of Plastic and Reconstructive Surgery, Incheon St. Mary's Hospital, College of Medicine, The Catholic University of Korea, 56 Dongsu-ro, Bupyeonggu, Incheon 21431, Korea

E-mail: ps.jylee@catholic.ac.kr

The abstract was presented at the PRS Korea 2019 in a poster session on November 8, 2019, in Seoul, Korea.

Received January 3, 2020 / Revised May 4, 2020 / Accepted June 19, 2020 to cicatricial alopecia [3]. Skin grafting is appropriate for larger defects, but skin grafts are too friable to be exposed without the protection of clothes; furthermore, the scar can be so wide that it is easily noticeable, and usually without hair [4]. Free flaps poorly match the scalp in terms of texture and contour and can result in loss of sensation. In contrast, tissue expansion offers aesthetically pleasing results and the maintenance of sensation with minimal donor-site morbidity. However, in a scarred scalp, the extent of possible dissection for expander insertion may be limited, since scars are composed of fibrous tissue with a different collagen alignment from that of normal tissue. As a result, surgeons must consider the nature of both the normal and scarred tissue in specific cases in order to achieve adequate expansion. We present two cases of scarred scalp defects that were successfully reconstructed with rectangular expanders. The publication of both cases was approved by the hospital institutional review board (IRB No. OC19ZISI0157). 


\section{CASE REPORTS}

\section{Case 1}

A 37-year-old woman presented with ongoing wound problems in the left temporoparietal region after surgery to remove a brain tumor and two radiation therapy sessions. Upon lysis of the eschar in the wound closure, the skull bone was exposed in a $4 \times 3 \mathrm{~cm}$ open wound. The wound margin was contractive with widespread scarring (Fig. 1A). Osteomyelitis was diagnosed based on culture results and was treated with 2 weeks of teicoplanin, 2 weeks of combination therapy (rifampicin with trimethoprim and sulfamethoxazole), and 2 weeks of linezolid. Reconstruction was performed using an expander. The incision line was designed on the right side of the scalp to avoid a chronic open wound. The pocket for the expander was planned in the frontoparietal region, considering future flap coverage. A dissection was made in the subgaleal plane, and a $400 \mathrm{~mL}$ smooth rectangular tissue expander was inserted. Since the defect was chronic and accompanied by osteomyelitis, there was a high risk of infection. Therefore, perioperative antibiotics were maintained for a week. Initial inflation was conducted 2 weeks after surgery to confirm the stability of the incision wound. The expander was inflated by a total of $195 \mathrm{~mL}$ over 2 months (Fig. 1B). Although radiation therapy and irregular scarring caused
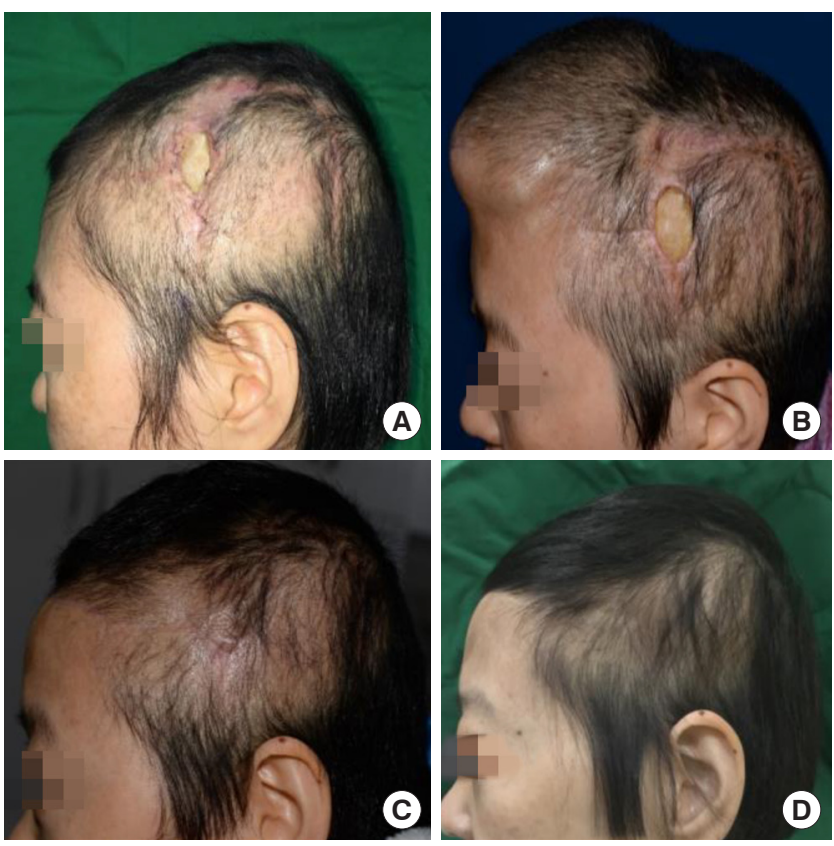

Fig. 1. A 37-year-old woman with a $4 \times 3 \mathrm{~cm}$ scalp defect. (A) The defect was accompanied by chronic unhealed scar tissue and osteomyelitis following a brain tumor removal operation. (B) A rectangular tissue expander was inserted in the subgaleal plane and was inflated by $195 \mathrm{~mL}$ over 2 months. (C) Three-month postoperative photograph. (D) Eighteen-month postoperative photograph. uneven expansion and thinning of the frontal scalp, we were able to achieve sufficient expansion to cover the defect despite only inflating the expander halfway. Two weeks after the final inflation, expander removal surgery was performed. A rotational fasciocutaneous flap was used to cover the defect.

\section{Case 2}

A 41-year-old woman had a $10 \times 15 \mathrm{~cm}$ bone-depth avulsion wound on the right parietal region of her scalp as a result of a degloving injury, as her hair had been sucked into a roller. Emergent microanastomosis was performed, but replantation of the avulsed scalp failed. Necrosis occurred thereafter. Debridement and a split-thickness skin graft were performed 1 month after the initial surgery. Scalp reconstruction with a tissue expander was planned, as a wide area of alopecia was noticeable (Fig. 2A). Ten months after the skin graft, the pocket for the expander was planned in the vertex region and an incision was made just medial to the scar tissue of the skin graft. A $400 \mathrm{~mL}$ smooth rectangular tissue expander was inserted in the subgaleal plane and immediately inflated by $20 \mathrm{~mL}$. During the dissection, firm adhesion and scarring were found throughout the subgaleal layer, making it difficult to dissect the tissue sufficiently. It was suspected that the galea was detached from the periosteum, which is a characteristic finding of degloving injuries.
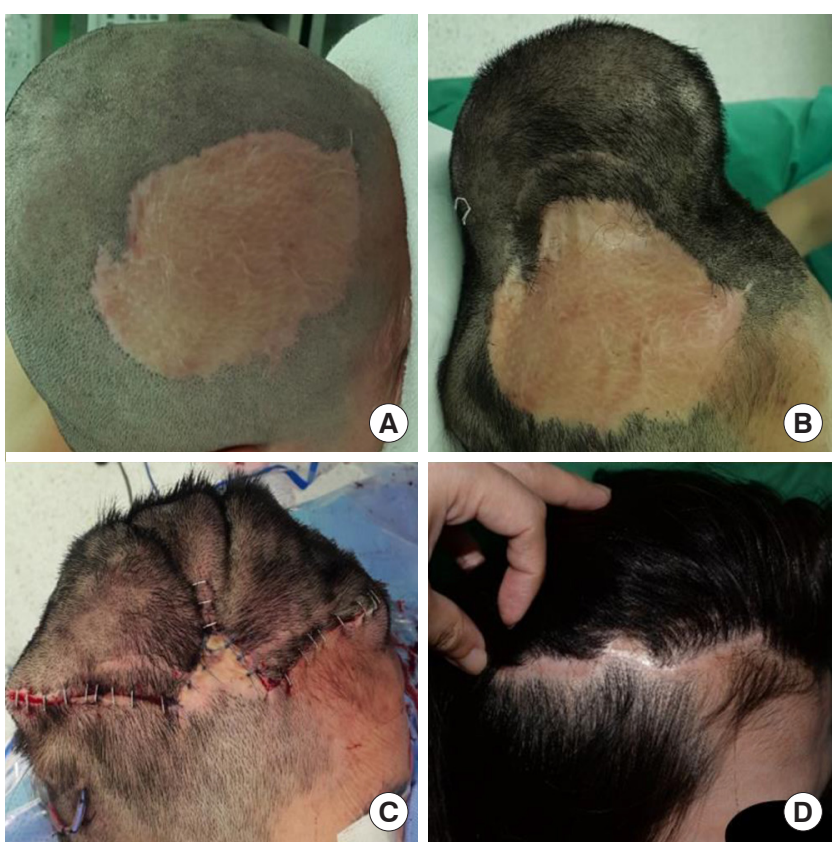

Fig. 2. A 41-year-old woman with an $11 \times 7.5 \mathrm{~cm}$ post-graft scar in the right frontoparietal region. (A) Preoperative view. (B) A rectangular tissue expander is inserted in the subgaleal plane and is inflated by 400 $\mathrm{mL}$ over 3 months. (C) The expanded tissue used as the advancement flap and the rotated flaps from the anterior and posterior base covered the lesion. (D) Twenty-four-month postoperative photograph. 
The expander was inflated 10 times by a total of $400 \mathrm{~mL}$ over the course of 3 months (Fig. 2B). Two months after the final inflation, the expander was removed and scar bands were scored to ensure better flap advancement. The anterior and posterior base was additionally undermined and a fasciocutaneous flap was used to cover the defect (Fig. 2C).

Both defects were well covered with good color, texture, and hair-bearing tissue (Figs. 1C and D, 2D). There were no complications involving tissue expanders, such as expander exposure, malfunction, rupture, infection, or hematoma. However, particularly thin skin at the vertex of the rectangular expander was noticeable.

\section{DISCUSSION}

Tissue expansion causes temporary epidermal thickening and thinning of the dermis. The expansion of the skin results from new creation of skin tissue by increased mitosis, rather than recruitment from an adjacent area [5]. Hair follicles neither increase in number nor degenerate, but become dispersed. However, this was not strikingly noticeable in our cases, even in large areas. Scalp reconstruction with tissue expansion inevitably affects the cranial bone because the scalp is a thin layer of tissue covering the bone. Tissue expansion has been found to decrease the thickness and volume of the cranial bone, but not bone density during expansion [6], but bone remodeling is completed between 2 and 3 months after the removal of the expander. In our cases, obtrusive bone loss or depression was not observed. Furthermore, it has been proven that the vascularity of expanded tissue increases [7], which contributes to the survival of flaps using expanded tissue, even when a large defect is being reconstructed. This is why tissue expansion can be recommended regardless of the size of the defect. Furthermore, tissue expansion is ideal for reconstructing the scalp because of the presence of hair-bearing tissue, which is not replaceable by distant tissues. For this reason, tissue expansion is used to cover areas of alopecia [8]. Caution should be taken when applying tissue expanders in the scalp to ensure that the expanders are not subject to pressure during sleep. In addition, the capsule and galea should be maximally preserved to maintain sufficient blood supply.

The incision site for the insertion of expanders should be carefully considered. When an incision is made in scarred tissue, it is preferable to avoid additional scarring by discarding the scarred tissue after obtaining the expanded tissue. However, the incision should only be made if the scar is mature and thick, in order to prevent extrusion of the expanders. Furthermore, dissection of the scarred wound in order to insert the expander is often challenging. Therefore, we recommend making the incision in normal tissue instead of in the fibrinous scar tissue. In case 1 , to reduce the possibility of infection and to facilitate dissection, an incision was made in normal tissue along the frontotemporal line. In case 2 , an incision was made in normal tissue, just medial to the scar tissue, due to the immature scar border and the high risk of extrusion of the expander. Although the tissue bridge between the scar tissue and the incision could develop necrosis, it was narrow enough for the expanded tissue to sufficiently replace it.

The subcutaneous and subgaleal layers have been recommended as planes for expander insertion in the scalp. We recommend the subgaleal plane to prevent extrusion, as the scalp tissue is thin. In addition, as the insertion plane can be easily dissected for the flap to be used as coverage after removing the expander, the future flap should be considered. Since a fasciocutaneous flap is better for the protection of exposed bone than a flap without the galea, the subgaleal plane was chosen. The initial inflation of the expander can be performed immediately to prevent seroma. However, if there is surrounding scar tissue, the contractile force can create pressure on the expander. The initial inflation and expansion velocity should be set in consideration of the area of the dissected space and the severity of scarring.

Tissue expansion affects the surrounding tissue in three dimensions. After expander removal, tissue deflates, which can be excessive when converted into two dimensions [9]. In this sense, rectangular expanders yield more available tissue than round or crescent-shaped expanders, assuming the same base area. Several mathematical formulas have been proposed for estimating the expansion volume to achieve a target surface area gain [10-12]. A study found that the surface area gain was $25 \%$ of the calculated value for spherical expanders and $38 \%$ and $32 \%$ with rectangular and crescent-shaped expanders, respectively [12]. Rectangular expanders are particularly effective for scar tissue, which requires more surface gain due to its contractile force. In addition, given the difficulty of dissecting scar tissue, it is necessary to maximize surface expansion with the least volume possible. As this study only presents two cases, it is difficult to present a generalized recommendation on expander insertion. However, it is noteworthy that the rectangular expander, when used on scarred wounds, secured an ample surface area without complications or bone resorption.

\section{NOTES}

\section{Conflict of interest}

No potential conflict of interest relevant to this article was reported. 


\section{Ethical approval}

The study was approved by the Institutional Review Board of Incheon St. Mary's Hospital (IRB No. OC19ZISI0157) and performed in accordance with the principles of the Declaration of Helsinki. Written informed consents were obtained.

\section{Patient consent}

The patients provided written informed consent for the publication and the use of their images.

\section{ORCID}

Hyojeong Shin https://orcid.org/0000-0002-1408-9013

Jeonghwan Shin https://orcid.org/0000-0001-9752-8941

Jun Yong Lee https://orcid.org/0000-0002-6967-4447

\section{REFERENCES}

1. Turko A, Fuzaylov G, Savchyn V, Driscoll D. Immediate and early tissue expander placement for acute closure of scalp wounds. Ann Plast Surg 2013;71:160-5.

2. Newman MI, Hanasono MM, Disa JJ, Cordeiro PG, Mehrara BJ. Scalp reconstruction: a 15-year experience. Ann Plast Surg 2004;52:501-6.

3. Belmahi A. The secondary surgeries after unfavorable results in scalp defects treatment. Ann Chir Plast Esthet 2019;64:54051.

4. Denewer A, Khater A, Farouk O, Hegazy M, Mosbah M, Hafez
$\mathrm{M}$, et al. Can we put a simplified algorithm for reconstruction of large scalp defects following tumor resection? World J Surg Oncol 2011;9:129.

5. Agrawal K, Agrawal S. Tissue regeneration during tissue expansion and choosing an expander. Indian J Plast Surg 2012; 45:7-15.

6. Moelleken BR, Mathes SJ, Cann CE, Simmons DJ, Ghafoori G. Long-term effects of tissue expansion on cranial and skeletal bone development in neonatal miniature swine: clinical findings and histomorphometric correlates. Plast Reconstr Surg 1990;86:825-34.

7. Marks MW, Burney RE, Mackenzie JR, Knight PR. Enhanced capillary blood flow in rapidly expanded random pattern flaps. J Trauma 1986;26:913-5.

8. Guzey S, Alhan D, Sahin I, Aykan A, Eski M, Nisancı M. Our experiences on the reconstruction of lateral scalp burn alopecia with tissue expanders. Burns 2015;41:631-7.

9. Hudson DA. Maximising the use of tissue expanded flaps. Br J Plast Surg 2003;56:784-90.

10. Pamplona DC, Weber HI, Leta FR. Optimization of the use of skin expanders. Skin Res Technol 2014;20:463-72.

11. Joss GS, Zoltie N, Chapman P. Tissue expansion technique and the transposition flap. Br J Plast Surg 1990;43:328-33.

12. van Rappard JH, Molenaar J, van Doorn K, Sonneveld GJ, Borghouts JM. Surface-area increase in tissue expansion. Plast Reconstr Surg 1988;82:833-9. 